\title{
Influencia del Estado de Madurez sobre las Propiedades Viscoelásticas de Frutas Tropicales (Mango, Papaya y Plátano)
}

\author{
Ramiro Torres, Everaldo J. Montes, Omar A. Pérez y Ricardo D. Andrade \\ Universidad de Córdoba, Grupo de Investigación GIPPAL, Facultad de Ingenierías, \\ Depto. de Ingeniería de alimentos, Km 12 Vía Cereté-Ciénaga de Oro-Colombia \\ (e-mail: rtorres@correo.unicordoba.edu.co)
}

Recibido Ene. 31, 2012; Aceptado Mar. 20, 2012; Versión final recibida Abr. 10, 2012

\section{Resumen}

En esta investigación se determinó la relación del estado de madurez con la viscoelasticidad, en mango de las variedades puerco y corazón, papaya de la variedad hawaiana y plátano. El estado de madurez se determinó por el color de la fruta visualmente, de acuerdo a una escala de maduración, y las propiedades viscoelásticas se determinaron por prueba de relajación y compresión del esfuerzo en un texturómetro. Dos modelos comunes, Maxwell generalizado y Peleg, se ajustaron a los datos experimentales. El modelo de Maxwell describió mejor el comportamiento viscoelástico de las frutas estudiadas. Los mangos de ambas variedades presentaron el mismo comportamiento viscoelástico en los diferentes estados de madurez, mientras que para papaya y plátano, el comportamiento elástico disminuyó con el estado de madurez.

\section{Influence of Maturity States on Viscoelastic Properties of Tropical Fruits (Mango, Papaya y Plantain)}

\begin{abstract}
In this investigation the relation of maturity states with viscoelasticity in two mango varieties (puerco and corazón), hawaiian papaya and plantain, were determined. Maturity state was determined by visually examining the color of the fruit, according to a maturation scale. Viscoelastic properties were determined by stress compression-relaxation tests using a texturometer. Two common models, generalized Maxwell and Peleg, were fitted to the experimental data. For all fruits, Maxwell model better described the viscoelastic behavior of the fruits. The two mango varieties showed the same viscoelastic behavior at different maturity states, while for papaya and banana, the elastic behavior decreased with maturity state.
\end{abstract}

Keywords: maturity states, viscoelastic, relaxation tests, Maxwell, Peleg 


\section{INTRODUCCIÓN}

La producción mundial de frutas tropicales en el año 2010 fue de aproximadamente 62 millones de toneladas, y se estima que para el 2014 la producción ascenderá a 82,1 millones de toneladas, lo que representa un incremento anual del 1,7 por ciento con respecto al período 2002-2004. Los países en desarrollo presentan un $98 \%$ de la producción. Se estima que América Latina y el Caribe representarán prácticamente todo el crecimiento de la producción mundial de frutas tropicales. El mango es la principal variedad de fruta tropical que se produce en todo el mundo, seguido de la piña, papaya, aguacate, guayaba y maracuyá. Según las proyecciones, la producción de mango será de 28,8 millones de toneladas en 2014 , lo que representará el $35 \%$ de la producción mundial de frutas tropicales, y América Latina participará con un $14 \%$ de la producción (FAO, 2012). En Colombia, la producción de frutas pasó de 6,47 a 7,52 millones de toneladas entre 2000 y 2009, correspondiente a un variación de 16,3\%, donde la papaya presentó el mayor crecimiento con una variación del $114,7 \%$; seguida del mango con un $77,1 \%$ y el banano con un $27,8 \%$ (Agronet, 2011).

Las pérdidas poscosecha de frutas tropicales varían entre un 10 y $80 \%$. En Colombia, solo por comercialización y transporte, estas pérdidas están alrededor del 10\% (Ciro et al., 2008). Las causas principales de las pérdidas poscosecha de los cultivos tropicales son, entre otros, los daños mecánicos causados por una mala manipulación a lo largo de la cadena del suministro; pérdida de humedad, envejecimiento prematuro por inadecuadas condiciones de almacenamiento, transporte a los centros de distribución, inexistencia de tecnologías de manejo y conservación y sobre todo poca industrialización (FAO, 2005). Los daños mecánicos pueden causar alteraciones metabólicas y fisiológicas, dando una apariencia anormal externa o interna y alteraciones en sabor y textura (Moretti y Sargent, 2000), afectando significativamente la composición fisicoquímica de los frutos (Casierra y Aguilar, 2008).

En condiciones de almacenamiento y procesamiento de alimentos, las propiedades mecánicas y reológicas constituyen un aspecto importante de calidad, especialmente las relacionadas con el esfuerzo y el tiempo que podrían soportar los alimentos antes de llegar a sus límites de elasticidad, de deformación plástica, o de resistencia mecánica en sus diferentes estados de madurez. La información reológica de los alimentos juega un papel primordial en su procesamiento, distribución y consumo; es importante para el desarrollo de procesos mecanizados de cosecha, en el diseño de procesos de transformación en alimentos; para la adopción, diseño y optimización de procesos de transformación, sistemas de empaque, transporte, manejo del producto, almacenamiento, y control de daño mecánico; en la determinación de la funcionalidad de los ingredientes para el desarrollo de productos y en el mejoramiento de los existentes; en el control de calidad de productos intermedios y finales; en pruebas de tiempo de vida útil y en evaluaciones de propiedades texturales correlacionadas con pruebas sensoriales (Ciro y Osorio, 2008; Rao et al., 2005).

Existen pocos trabajos relacionados con la caracterización reológica de frutas tropicales, entre los cuales se destacan los relacionados con determinación de la fuerza de fractura y firmeza en frutas tropicales como uchuva (Ciro y Osorio, 2008), lulo (Ospina et al., 2007), tomate de árbol (Ciro et al., 2005), yuca (Linares et al., 2005) y plátano (Ciro et al., 2008). En Colombia existe un escaso conocimiento del comportamiento reológico de la mayoría de los productos agropecuarios. El objetivo de este trabajo de investigación fue determinar la influencia del estado de madurez sobre las propiedades viscoelásticas del mango, papaya y plátano. $\square$

\section{MATERIALES Y METODOS}

\section{Materia prima}

Se utilizaron frutos de mango (Mangifera indica $L$ ) variedad puerco y corazón, papaya (Carica papaya L.) variedad Hawaiana y plátano (Mussa Cavendish), provenientes de Montería (Colombia) en diferentes estados de madurez, los cuales fueron identificados visualmente por el 
color de la cáscara mediante la escala de maduración para mango (Peña, 2008), papaya (Santamaría et al., 2009) y plátano (Dadzie y Orchard, 1997).

\section{Estados de madurez (EM)}

Se determinaron los rangos de color para los EM clasificados tradicionalmente, teniendo en cuenta el valor máximo de diferencia de color (5,6 unidades) entre sí, lo cual se considera como una tolerancia normal perceptible para el ojo humano (Melgosa et al., 2001). Cada EM se representó como un patrón o estándar, enumerándolos del 1 al 7, para papaya y plátano y del 1 al 6 para las variedades de mango.

Prueba de relajación

Se realizaron pruebas de relajación en mango, papaya y plátano, en compresión uníaxial sobre muestras cilíndricas de $2 \mathrm{~cm}$ de diámetro y $3 \mathrm{~cm}$ de altura, mediante el plato de compresión de 50 $\mathrm{mm}$ de diámetro a una velocidad de $1 \mathrm{~mm} / \mathrm{s}$, hasta $10 \%$ respecto a la altura inicial, y un tiempo de relajación de 1200 s. Los ensayos se realizaron con un Texturómetro Shimadzu EZ Test ${ }^{\circledR}$ (Shimadzu Corporation, Japón), usando el software RheoMeter ${ }^{\circledR}$ Versión 2.04.

A partir de las curvas fuerza-tiempo, se calcularon la deformación real, $\varepsilon(t)$ y esfuerzo real, $\sigma(t)$, en función del tiempo, utilizando las ecuaciones (1) y (2), respectivamente.

$$
\begin{aligned}
& \varepsilon(\mathrm{t})=-\int_{\mathrm{h}_{0}}^{\mathrm{h}} \frac{\mathrm{dh}}{\mathrm{h}} \quad \varepsilon(\mathrm{t})=\ln \frac{\mathrm{h}_{0}}{h(t)}=\ln \frac{\mathrm{h}_{0}}{h_{0}-\Delta h(\mathrm{t})} \\
& \sigma_{\mathrm{E}}(\mathrm{t})=\frac{\mathrm{F}(\mathrm{t})}{\mathrm{A}_{0}} \\
& \sigma(\mathrm{t})=\frac{\mathrm{F}(\mathrm{t})}{A(\mathrm{t})}=\frac{F(\mathrm{t})\left[\mathrm{h}_{0}-\Delta \mathrm{h}(\mathrm{t})\right]}{A_{0} \mathrm{~h}_{0}}
\end{aligned}
$$

Donde $h_{0}$ y $h(t)$ es la longitud de la muestra al inicio y al tiempo $t$, respectivamente; $F(t)$ es la fuerza ejercida a la muestra en el tiempo y $A_{0}$ y $A(t)$ es el área inicial y real en el tiempo $t$, respectivamente. Con la deformación real y esfuerzo real se determinó el módulo de relajación, $E(t)$, en función del tiempo, el cual fue normalizado, $Y(t)$, para corregir las diferencias de magnitud inicial del esfuerzo requerido para deformar la muestra hasta el $10 \%$ de deformación. Los datos experimentales se ajustaron al modelo de Maxwell generalizado con 3 términos (Ecuación 3), por regresión no lineal utilizando el algoritmo de Levenberg-Marquardt, obteniéndose los parámetros del modelo $\left(E_{1}, E_{2}, E_{3} \tau_{1}\right.$ y $\left.\tau_{2}\right)$ y los parámetros $S^{*}$ (Ecuación 4), relacionado con velocidad inicial de relación y $\mathrm{P}^{\star}$ (Ecuación 5), valor residual asintótico, sugeridos por Bellido y Hatcher (2009).

$$
\begin{aligned}
& \mathrm{E}(\mathrm{t})=\mathrm{E}_{1} \mathrm{e}^{-\frac{\mathrm{t}}{\tau 1}}+E_{2} e^{-\frac{\mathrm{t}}{\tau 2}}+E_{3} \\
& \mathrm{~S}=\int_{0}^{\mathrm{t}} \mathrm{Y}(\mathrm{t}) \mathrm{dt} \\
& \mathrm{P}=\frac{\mathrm{P}_{0}}{\mathrm{P}(0)}
\end{aligned}
$$

Los datos de relajación fueron ajustados al modelo empírico de Peleg (Ecuación 6), por regresión lineal basado en el algoritmo de Levenberg-Marquardt, obteniendo los parámetros a (nivel de caída del esfuerzo) y b (velocidad inicial de relajación). Además el parámetro Ea (modulo asintótico residual) fue determinado según la ecuación (7). La validez de los modelos se evalúo 
utilizando los parámetros estadísticos de coeficiente de determinación y el análisis de los residuales del modelo.

$\frac{\mathrm{t}}{\mathrm{Y}(\mathrm{t})}=\frac{1}{\mathrm{ab}}+\frac{\mathrm{t}}{\mathrm{a}}$

$\mathrm{E}_{\mathrm{a}}=\mathrm{E}_{0}(1-\mathrm{a})$

\section{Análisis estadístico}

El experimento, para la el mango, fue conducido bajo un diseño en bloque completamente al asar (DBCA), unifactorial (estado de madurez) en 6 niveles $(1,2,3,4,5$ y 6 ), bloqueando el factor variedad (puerco y corazón) para las variables respuestas: esfuerzo y deformación unitaria, con tres réplicas. El experimento para papaya y plátano, fue conducido bajo un diseño completamente al asar (DCA), con factores de efectos principales y de efecto fijos, el factor estado de madurez en 7 niveles, y el factor sentido de la carga en 2 niveles (transversal y perpendicular a la dirección de la fibra), para las variables respuestas: esfuerzo y deformación unitaria, con 3 repeticiones. Se realizó un ANOVA a un nivel de significancia al 5\% y una prueba de comparación de medias. El ANOVA e interpretación estadística se realizó con el Software STATISTICA ${ }^{\circledR} 8.0$ (StatSoft, Inc., 2007) y el ajuste de la curva con el software OriginPro ${ }^{\circledR} 8.0$ (OriginLab Corporation, 2007).

\section{RESULTADOS Y DISCUSIONES}

El comportamiento de relajación para el mango variedad puerco ajustado a los modelos de Maxwell y Peleg se muestra en la Figura 1. Igual comportamiento se presentó para papaya y plátano. A pesar de la obvia facilidad del manejo del modelo de Peleg las gráficas muestran que las predicciones teóricas derivadas de este modelo no describen exactamente los datos experimentales, aunque se presenten coeficientes de determinación muy cercanos a $1\left(R^{2}>\right.$ 0.991). El ajuste lineal con respecto a los datos experimentales en los primeros $10 \mathrm{~s}$, presenta una desviación que es significativa, además en esta zona es donde se dan las mayores velocidades de decaimiento. Esto concuerda con lo reportado para salchichas (Andrés et al., 2008) y tallarines (Bellido y Hatcher, 2009).
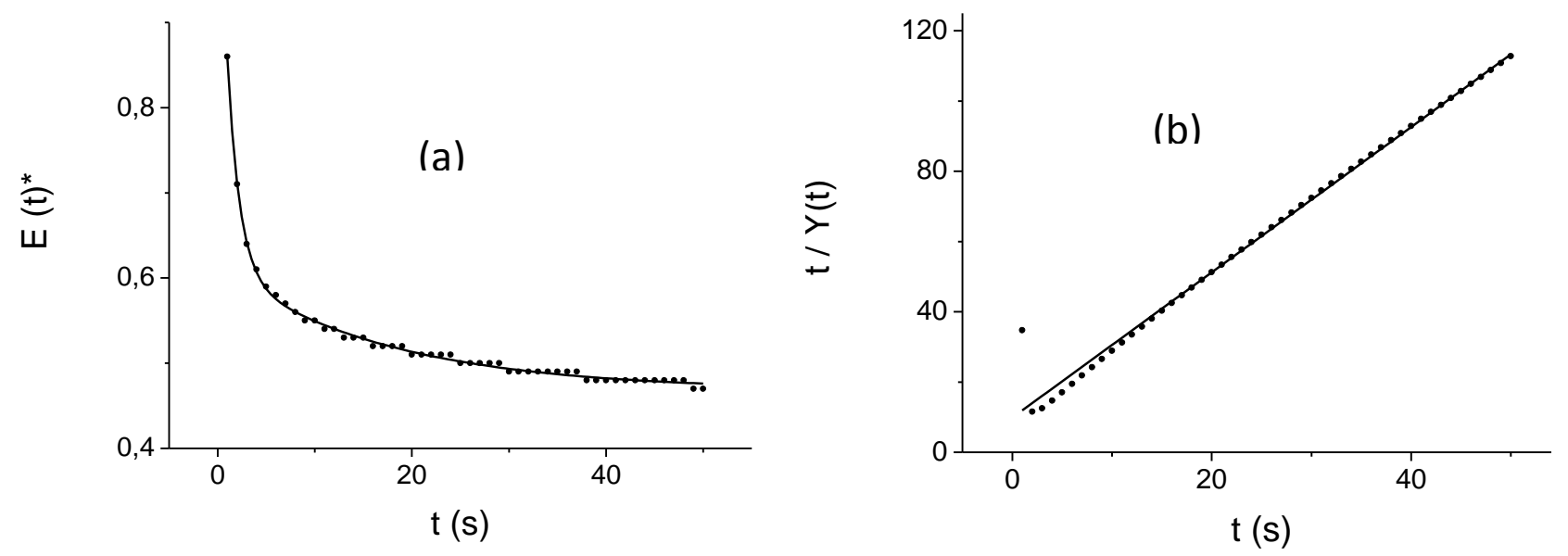

Fig. 1: Prueba de relajación para mango variedad puerco, modelo de Maxwell (a) y Peleg (b)

El análisis de residuales (prueba de normalidad de Shapiro-Wilk), para todas las frutas evaluadas en cada uno de los estados de madurez mostró que el modelo de Maxwell presentó una distribución normal, cosa contraria al modelo de Peleg. Esto indica que los datos experimentales de relajación de las frutas estudiadas, pueden ser representado por el modelo de Maxwell y se podrían aproximar al modelo de Peleg. Esto se debe a que el ajuste lineal, causa que los datos situados en el extremo final de la curva de relajación ejerzan una mayor influencia sobre b y a. Los datos en esta porción de la curva constituyen más de $75 \%$ de todos los datos. En consecuencia, b 
representa muy poco la velocidad inicial de relajación de los alimentos, particularmente para alimentos cuya respuesta de relajación decae rápidamente, por tanto el modelo de Peleg no puede explicar componentes de relajación con tiempo de relajación corto.

La velocidad inicial de relajación (velocidad de relajación rápida), es representada por $\tau_{2}$, ya que tiene valores menores que $\tau_{1}$ (Tablas 1,2 y 3). La velocidad de relajación inicial aumenta con el estado de madurez, variación representada sólo por el parámetro $E_{2}$, ya que $\tau_{2}$ no presenta diferencias significativas entre éstos. Por otra parte, la velocidad de relajación lenta, para diferentes estados de madurez, es sólo influenciada por el parámetro $\tau_{1}$ ya que $E_{1}$ no presenta diferencias significativas entre los estados de madurez. El parámetro $\mathrm{E}_{3}$ que es el valor asintótico de relajación, disminuyó a medida que aumenta el estado de madurez, indicando que la elasticidad de las frutas aumenta a medida que aumenta el estado de madurez.

Tabla 1: Parámetros del modelo de Maxwell para el mango a diferentes EM y variedades (V). ( ${ }^{*}$ Letras diferentes indican diferencias significativas en comparación de medias para EM).

\begin{tabular}{|c|c|c|c|c|c|c|c|}
\hline V & EM & $\mathrm{E}_{1}(\mathrm{kPa})$ & $\mathrm{E}_{2}(\mathrm{kPa})$ & $\mathrm{E}_{3}(\mathrm{kPa})$ & $\tau_{1}(\mathrm{~s})$ & $\tau_{2}(\mathrm{~s})$ & $\mathrm{R}^{2}$ \\
\hline \multirow{6}{*}{$\frac{\frac{O}{0}}{\frac{\Phi}{2}}$} & 1 & $0,21^{b^{*}} \pm 0,005$ & $0,13^{\mathrm{c}} \pm 0,008$ & $0,55^{c} \pm 0,005$ & $23,67^{\mathrm{C}} \pm 2,09$ & $2,70^{a} \pm 0,34$ & 0,9973 \\
\hline & 2 & $0,24^{b} \pm 0,004$ & $0,19^{b c} \pm 0,007$ & $0,48^{b c} \pm 0,004$ & $21,47^{\mathrm{bc}} \pm 1,15$ & $2,12^{a} \pm 0,17$ & 0,9984 \\
\hline & 3 & $0,22^{b} \pm 0,006$ & $0,23^{\mathrm{abc}} \pm 0,014$ & $0,45^{a b c} \pm 0,003$ & $17,44^{\mathrm{abc}} \pm 1,12$ & $1,66^{a} \pm 0,18$ & 0,9963 \\
\hline & 4 & $0,31^{b} \pm 0,006$ & $0,25^{\mathrm{abc}} \pm 0,014$ & $0,43^{\mathrm{abc}} \pm 0,003$ & $12,56^{\mathrm{abc}} \pm 1,12$ & $1,75^{a} \pm 0,18$ & 0,9962 \\
\hline & 5 & $0,24^{b} \pm 0,006$ & $0,59^{\mathrm{ab}} \pm 0,021$ & $0,34^{\mathrm{ab}} \pm 0,003$ & $14,90^{\mathrm{ab}} \pm 0,78$ & $1,32^{a} \pm 0,07$ & 0,9976 \\
\hline & 6 & $0,10^{a} \pm 0,004$ & $0,86^{a} \pm 0,020$ & $0,25^{a} \pm 0,002$ & $15,55^{\mathrm{a}} \pm 1,47$ & $1,20^{a} \pm 0,04$ & 0,9977 \\
\hline \multirow{6}{*}{ 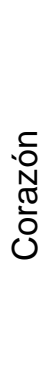 } & 1 & $0,15^{a} \pm 0,003$ & $0,41^{a} \pm 0,014$ & $0,57^{\mathrm{a}} \pm 0,003$ & $20,35^{a} \pm 1,517$ & $1,45^{a} \pm 0,073$ & 0,9970 \\
\hline & 2 & $0,17^{a} \pm 0,004$ & $0,29^{a} \pm 0,010$ & $0,57^{a} \pm 0,003$ & $19,96^{\mathrm{ab}} \pm 1,375$ & $1,77^{\mathrm{a}} \pm 0,105$ & 0,9975 \\
\hline & 3 & $0,23^{a} \pm 0,006$ & $0,50^{a} \pm 0,026$ & $0,45^{\mathrm{ab}} \pm 0,002$ & $12,36^{\mathrm{abc}} \pm 0,517$ & $1,10^{\mathrm{a}} \pm 0,072$ & 0,9975 \\
\hline & 4 & $0,21^{a} \pm 0,004$ & $0,36^{a} \pm 0,013$ & $0,41^{\mathrm{ab}} \pm 0,003$ & $17,39^{\mathrm{abc}} \pm 0,892$ & $1,50^{\mathrm{a}} \pm 0,088$ & 0,9978 \\
\hline & 5 & $0,19^{a} \pm 0,004$ & $0,70^{\mathrm{a}} \pm 0,028$ & $0,38^{\mathrm{ab}} \pm 0,002$ & $16,45^{b c} \pm 0,868$ & $1,04^{a} \pm 0,046$ & 0,9976 \\
\hline & 6 & $0,13^{a} \pm 0,004$ & $0,62^{a} \pm 0,014$ & $0,35^{\mathrm{b}} \pm 0,003$ & $18,41^{\mathrm{C}} \pm 1,538$ & $1,46^{a} \pm 0,050$ & 0,9978 \\
\hline
\end{tabular}

EI ANOVA para los parámetros $S^{*}$ y b muestra que hay diferencias significativas entre los estados de madurez en el mango variedad puerco y corazón (Tabla 4), al igual que en la papaya (Tabla 5) y plátano (Tabla 6), es decir que la velocidad inicial de relajación presenta diferencias significativas entre los estados de madurez; sin embargo en el mango no existen diferencias significativas entre las variedades, al igual que el sentido de carga para la papaya. En el plátano se presentan diferencias significativas en el tratamiento sentido de carga, indicando que es una estructura anisotrópica, lo cual concuerda con resultados reportados por Ciro et al. (2008).

El test de comparación de medias (Tukey) para $S^{*}$ para mango variedad puerco y corazón (Tabla 4), muestra que $S^{*}$ disminuye (velocidad inicial de relajación aumenta) con en el estado de madurez, aunque en el parámetro $b$ no se presentaron diferencias significativas (a una significancia del $5 \%$ ). El test de comparación de medias (Tukey) para papaya (Tabla 5) y plátano (Tabla 6) muestra que $S^{*}$ disminuye y b aumenta (velocidad inicial de relajación aumenta) con el estado de madurez. Además para el plátano la velocidad de relajación fue mayor en el sentido de carga longitudinal que transversal. El parámetro $S^{*}$ es más eficaz para representar adecuadamente la velocidad inicial de relajación de los datos experimentales que b. Esto es de esperase ya que $S^{*}$, fue derivado de un modelo que describió fielmente la respuesta de la relajación de los datos experimentales. 
Tabla 2: Parámetros de modelo de Maxwell para papaya a diferentes EM y sentido de carga (SC). ( ${ }^{*}$ Letras diferentes indican diferencias significativas en comparación de medias para EM).

\begin{tabular}{|c|c|c|c|c|c|c|c|}
\hline SC & EM & $\mathrm{E}_{1}(\mathrm{kPa})$ & $\mathrm{E}_{2}(\mathrm{kPa})$ & $\mathrm{E}_{3}(\mathrm{kPa})$ & $\tau_{1}(\mathrm{~s})$ & $\tau_{2}(\mathrm{~s})$ & $\mathrm{R}^{2}$ \\
\hline \multirow{7}{*}{ 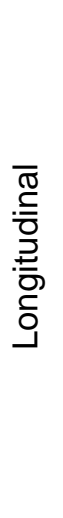 } & 1 & $0,17^{a^{*}} \pm 0,003$ & $0,12^{a} \pm 0,01$ & $0,62^{a} \pm 0,005$ & $25,94^{a} \pm 1,96$ & $1,60^{a} \pm 0,22$ & 0,9960 \\
\hline & 2 & $0,18^{a} \pm 0,004$ & $0,10^{a} \pm 0,01$ & $0,59^{\mathrm{a}} \pm 0,007$ & $26,14^{a} \pm 2,99$ & $1,50^{a} \pm 0,42$ & 0,9955 \\
\hline & 3 & $0,21^{a} \pm 0,004$ & $0,22^{b} \pm 0,01$ & $0,26^{b} \pm 0,004$ & $21,23^{b} \pm 1,34$ & $2,08^{a} \pm 0,15$ & 0,9980 \\
\hline & 4 & $0,21^{a} \pm 0,004$ & $0,25^{\mathrm{b}} \pm 0,01$ & $0,18^{b} \pm 0,004$ & $20,97^{b} \pm 1,34$ & $1,76^{a} \pm 0,13$ & 0,9974 \\
\hline & 5 & $0,17^{\mathrm{a}} \pm 0,004$ & $0,23^{b} \pm 0,02$ & $0,17^{b} \pm 0,003$ & $17,09^{b} \pm 1,02$ & $1,26^{a} \pm 0,14$ & 0,9955 \\
\hline & 6 & $0,17^{\mathrm{a}} \pm 0,004$ & $0,23^{b} \pm 0,02$ & $0,17^{b} \pm 0,003$ & $17,09^{b} \pm 1,02$ & $1,26^{a} \pm 0,14$ & 0,9955 \\
\hline & 7 & $0,20^{a} \pm 0,004$ & $0,24^{b} \pm 0,01$ & $0,16^{b} \pm 0,003$ & $18,62^{b} \pm 1,14$ & $1,55^{a} \pm 0,14$ & 0,9966 \\
\hline \multirow{7}{*}{ 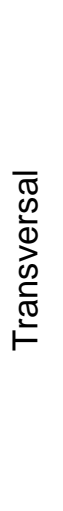 } & 1 & $0,18^{\mathrm{a}} \pm 0,004$ & $0,10^{a} \pm 0,01$ & $0,58^{\mathrm{a}} \pm 0,006$ & $24,81^{a} \pm 2,46$ & $2,54^{a} \pm 0,40$ & 0,9962 \\
\hline & 2 & $0,18^{a} \pm 0,004$ & $0,15^{a} \pm 0,01$ & $0,47^{\mathrm{ab}} \pm 0,006$ & $24,61^{a} \pm 2,55$ & $2,51^{a} \pm 0,28$ & 0,9964 \\
\hline & 3 & $0,22^{a} \pm 0,004$ & $0,43^{b} \pm 0,01$ & $0,35^{\mathrm{abc}} \pm 0,002$ & $14,19^{b} \pm 0,56$ & $0,64^{b} \pm 0,03$ & 0,9981 \\
\hline & 4 & $0,18^{\mathrm{a}} \pm 0,003$ & $0,44^{b} \pm 0,03$ & $0,24^{b c} \pm 0,002$ & $16,22^{b} \pm 0,75$ & $1,01^{b} \pm 0,06$ & 0,9972 \\
\hline & 5 & $0,20^{a} \pm 0,003$ & $0,22^{\mathrm{b}} \pm 0,01$ & $0,25^{b c} \pm 0,004$ & $22,57^{b} \pm 1,59$ & $1,93^{b} \pm 0,15$ & 0,9974 \\
\hline & 6 & $0,17^{\mathrm{a}} \pm 0,004$ & $0,24^{b} \pm 0,01$ & $0,20^{b c} \pm 0,003$ & $19,48^{b} \pm 1,51$ & $1,69^{b} \pm 0,15$ & 0,9960 \\
\hline & 7 & $0,18^{a} \pm 0,004$ & $0,10^{a} \pm 0,01$ & $0,58^{a} \pm 0,006$ & $24,81^{a} \pm 2,46$ & $2,54^{a} \pm 0,40$ & 0,9962 \\
\hline
\end{tabular}

EI ANOVA para los parámetros $P^{*}$ y a, en diferentes estados de madurez del mango variedad puerco y corazón (Tabla 4) muestra que hay diferencias significativas (significancia del $5 \%$ ) entre los estado de madurez en el mango variedad puerco, igual comportamiento presentaron la variedad corazón, la papaya y el plátano es decir, el estado de madurez incide sobre el comportamiento viscoelástico. En el mango, no existen diferencias significativas entre las variedades, al igual que el sentido de la carga para la papaya. En el plátano se presentan diferencias significativas en el tratamiento sentido de la carga, corroborando lo expuesto inicialmente que el plátano presenta una estructura anisotrópico.

El test de comparación de medias (Tukey) para en el mango variedad puerco y corazón (Tabla 4), al igual que en la papaya (Tabla 5) y plátano (Tabla 6) muestra que $\mathrm{P}^{*}$ disminuye y a aumenta con el estado de madurez, indicando que el esfuerzo aplicado durante la carga es disipado en mayor cantidad a estados de madurez mayor (el comportamiento elástico disminuye con el estado de madurez). Sin embargo, en los estados madurez intermedio (3, 4 y 6), el mango presentan igual comportamiento viscoelástico. Para la papaya y plátano los frutos más maduros (EM 3 al 7 ) presentan igual comportamiento viscoelástico. Respecto al sentido de la carga para el plátano, en el sentido longitudinal se disipó una menor cantidad de esfuerzo aplicado, es decir, tenían mayor comportamiento elástico que en el sentido transversal.

Esto es posible debido a que a, es influenciado por todo el conjunto de datos linealizados diferenciando mejor la respuesta relativa de la relajación de las frutas estudiadas, concordando con estudios recientes en tallarines (Hatcher et al., 2008, Hatcher et al., 2009) salchichas cocidas y cruda, pasta, gelatina, queso de soja, torta, queso mozarela, queso Cheddar, pastel de ángel y pan (Singh et al., 2006). 
Tabla 3: Parámetros de modelo de maxwell para plátano a diferentes EM y sentido de carga (SC). ( ${ }^{\star}$ Letras diferentes indican diferencias significativas, en comparación de medias para EM).

\begin{tabular}{|c|c|c|c|c|c|c|c|}
\hline SC & EM & $\mathrm{E}_{1}(\mathrm{kPa})$ & $\mathrm{E}_{2}(\mathrm{kPa})$ & $\mathrm{E}_{3}(\mathrm{kPa})$ & $\tau_{1}(\mathrm{~s})$ & $\tau_{2}(\mathrm{~s})$ & $\mathrm{R}^{2}$ \\
\hline \multirow{7}{*}{ 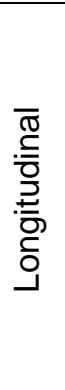 } & 1 & $0,17^{\mathrm{a}^{*}} \pm 0,006$ & $0,08^{a} \pm 0,01$ & $0,69^{a} \pm 0,011$ & $35,15^{a} \pm 5,16$ & $2,76^{a} \pm 0,43$ & 0,9959 \\
\hline & 2 & $0,15^{\mathrm{a}} \pm 0,006$ & $0,09^{a} \pm 0,01$ & $0,69^{a} \pm 0,011$ & $35,08^{a} \pm 6,22$ & $3,16^{\mathrm{a}} \pm 0,47$ & 0,9954 \\
\hline & 3 & $0,18^{\mathrm{a}} \pm 0,004$ & $0,15^{b} \pm 0,01$ & $0,47^{b} \pm 0,004$ & $21,50^{b} \pm 1,73$ & $2,52^{a} \pm 0,25$ & 0,9975 \\
\hline & 4 & $0,16^{a} \pm 0,005$ & $0,15^{\mathrm{b}} \pm 0,01$ & $0,46^{b} \pm 0,006$ & $23,56^{b} \pm 2,89$ & $2,88^{a} \pm 0,31$ & 0,9965 \\
\hline & 5 & $0,18^{\mathrm{a}} \pm 0,004$ & $0,15^{\mathrm{b}} \pm 0,01$ & $0,47^{b} \pm 0,004$ & $21,84^{b} \pm 1,88$ & $2,54^{a} \pm 0,26$ & 0,9975 \\
\hline & 6 & $0,15^{a} \pm 0,005$ & $0,17^{\mathrm{b}} \pm 0,01$ & $0,44^{b} \pm 0,005$ & $23,21^{b} \pm 2,89$ & $2,74^{a} \pm 0,27$ & 0,9963 \\
\hline & 7 & $0,18^{a} \pm 0,004$ & $0,18^{b} \pm 0,01$ & $0,42^{b} \pm 0,006$ & $23,81^{b} \pm 2,60$ & $2,34^{a} \pm 0,25$ & 0,9957 \\
\hline \multirow{7}{*}{ 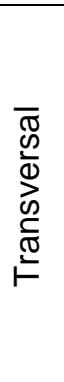 } & 1 & $0,16^{a} \pm 0,003$ & $0,11^{a} \pm 0,007^{*}$ & $0,65^{\mathrm{a}} \pm 0,005$ & $24,52^{\mathrm{a}} \pm 2,32$ & $2,21^{a} \pm 0,30$ & 0,9958 \\
\hline & 2 & $0,15^{a} \pm 0,010$ & $0,09^{a} \pm 0,004$ & $0,67^{\mathrm{a}} \pm 0,003$ & $31,25^{a} \pm 0,10$ & $3,95^{a} \pm 1,38$ & 0,9960 \\
\hline & 3 & $0,21^{a} \pm 0,004$ & $0,17^{b} \pm 0,009$ & $0,40^{b} \pm 0,007$ & $24,75^{a} \pm 2,43$ & $2,31^{a} \pm 0,26$ & 0,9963 \\
\hline & 4 & $0,19^{a} \pm 0,006$ & $0,16^{b} \pm 0,009$ & $0,41^{b} \pm 0,005$ & $21,62^{\mathrm{a}} \pm 2,21$ & $2,56^{a} \pm 0,32$ & 0,9962 \\
\hline & 5 & $0,17^{\mathrm{a}} \pm 0,005$ & $0,17^{b} \pm 0,009$ & $0,40^{b} \pm 0,008$ & $25,73^{\mathrm{a}} \pm 3,71$ & $2,87^{a} \pm 0,33$ & 0,9956 \\
\hline & 6 & $0,21^{a} \pm 0,004$ & $0,19^{b} \pm 0,008$ & $0,37^{b} \pm 0,006$ & $25,75^{\mathrm{a}} \pm 2,40$ & $2,45^{a} \pm 0,22$ & 0,9973 \\
\hline & 7 & $0,21^{a} \pm 0,004$ & $0,19^{b} \pm 0,008$ & $0,37^{b} \pm 0,006$ & $25,75^{\mathrm{a}} \pm 2,40$ & $2,45 \pm 0,22$ & 0,9973 \\
\hline
\end{tabular}

Tabla 4: Parámetros del modelo de Peleg y de Bellidio- Hatcher, para mango a diferentes EM y variedades $(\mathrm{V})$. ('Letras diferentes indican diferencias significativas, en comparación de medias para EM).

\begin{tabular}{|c|c|c|c|c|c|c|c|}
\hline \multirow{2}{*}{ V } & \multirow{2}{*}{ EM } & \multicolumn{2}{|c|}{ Modelo de Peleg } & \multicolumn{3}{|c|}{ Modelo de Maxwell } & \multirow{2}{*}{$\mathrm{R}^{2}$} \\
\hline & & $S^{*}$ & $P^{*}$ & $\mathrm{Ea}$ & a & $b$ & \\
\hline \multirow{6}{*}{$\begin{array}{l}\text { O্ } \\
\frac{\Phi}{2} \\
0\end{array}$} & 1 & $0,64^{\mathrm{c}} \pm 0,01$ & $0,54^{\mathrm{C}} \pm 0,02$ & $0,54^{b} \pm 0,01$ & $0,46^{b} \pm 0,01$ & $0,22^{b} \pm 0,01$ & 0,9968 \\
\hline & 2 & $0,57^{b c} \pm 0,08$ & $0,47^{b c} \pm 0,07$ & $0,46^{\mathrm{ab}} \pm 0,08$ & $0,54^{\mathrm{ab}} \pm 0,08$ & $0,23^{a} \pm 0,04$ & 0,9974 \\
\hline & 3 & $0,52^{\mathrm{abc}} \pm 0,06$ & $0,44^{\mathrm{abc}} \pm 0,05$ & $0,44^{\mathrm{ab}} \pm 0,05$ & $0,56^{\mathrm{ab}} \pm 0,05$ & $0,32^{a} \pm 0,03$ & 0,9989 \\
\hline & 4 & $0,52^{\mathrm{abc}} \pm 0,13$ & $0,42^{\mathrm{abc}} \pm 0,11$ & $0,40^{\mathrm{ab}} \pm 0,14$ & $0,60^{\mathrm{ab}} \pm 0,14$ & $0,27^{a} \pm 0,08$ & 0,9985 \\
\hline & 5 & $0,41^{\mathrm{ab}} \pm 0,08$ & $0,33^{\mathrm{ab}} \pm 0,05$ & $0,33^{\mathrm{ab}} \pm 0,00$ & $0,67^{\mathrm{ab}} \pm 0,00$ & $0,33^{a} \pm 0,13$ & 0,9996 \\
\hline & 6 & $0,29^{a} \pm 0,08$ & $0,25^{a} \pm 0,07$ & $0,28^{a} \pm 0,07$ & $0,72^{a} \pm 0,07$ & $0,70^{a} \pm 0,05$ & 0,9996 \\
\hline \multirow{6}{*}{ 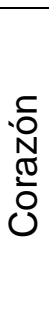 } & 1 & $0,63^{a} \pm 0,03$ & $0,57^{a} \pm 0,03$ & $0,58^{a} \pm 0,02$ & $0,42^{a} \pm 0,02$ & $0,20^{a} \pm 0,06$ & 0,9841 \\
\hline & 2 & $0,64^{a} \pm 0,07$ & $0,57^{\mathrm{a}} \pm 0,07$ & $0,57^{a} \pm 0,06$ & $0,43^{a} \pm 0,06$ & $0,24^{a} \pm 0,03$ & 0,9991 \\
\hline & 3 & $0,50^{a} \pm 0,07$ & $0,44^{\mathrm{ab}} \pm 0,06$ & $0,44^{\mathrm{ab}} \pm 0,07$ & $0,56^{\mathrm{ab}} \pm 0,07$ & $0,28^{a} \pm 0,04$ & 0,9994 \\
\hline & 4 & $0,49^{a} \pm 0,07$ & $0,41^{\mathrm{ab}} \pm 0,07$ & $0,41^{\mathrm{ab}} \pm 0,07$ & $0,59^{\mathrm{ab}} \pm 0,07$ & $0,33^{a} \pm 0,05$ & 0,9993 \\
\hline & 5 & $0,44^{a} \pm 0,20$ & $0,37^{\mathrm{ab}} \pm 0,16$ & $0,37^{\mathrm{ab}} \pm 0,15$ & $0,42^{\mathrm{ab}} \pm 0,38$ & $0,28^{a} \pm 0,30$ & 0,9992 \\
\hline & 6 & $0,41^{a} \pm 0,06$ & $0,35^{b} \pm 0,06$ & $0,36^{b} \pm 0,05$ & $0,64^{b} \pm 0,05$ & $0,49^{a} \pm 0,24$ & 0,9985 \\
\hline
\end{tabular}

Por ultimo, el modelo de Maxwell es capaz de describir adecuadamente el comportamiento de relajación de las frutas usando los parámetros, $S^{*}$ (forma de la función) y $\mathrm{P}^{*}$ (valor asintótico residual de la función), propuesto por Bellido y Hatcher (2009) sugiriendo que el método propuesto no es menos eficaz que el método de Peleg para facilitar la comparación de las curvas de relajación. $\mathrm{P}^{\star}$ y a fueron capaces de distinguir de igual manera el comportamiento viscoelástico de las muestras experimentales, y la relación entre las magnitudes de estas dos variables fueron directas según lo predicho por Peleg y Normand (1983): $P^{*}=1-a \quad\left(R^{2}=0,97\right)$, que corresponde con Ea. 
Tabla 5: Parámetros del modelo de Peleg y de Bellidio- Hatcher (modelo de Maxwell) a diferentes EM y sentido de carga (SC) para papaya. ${ }^{* *}$ Letras diferentes indican diferencias significativas en comparación de medias para EM)

\begin{tabular}{|c|c|c|c|c|c|c|c|}
\hline SC & EM & $\mathrm{S}^{*}$ & $\mathrm{P}^{*}$ & $\mathrm{Ea}$ & $\mathrm{a}$ & $\mathrm{b}$ & $\mathrm{R}^{2}$ \\
\hline \multirow{7}{*}{ 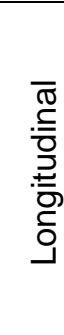 } & 1 & $0,70^{\mathrm{a}} \pm 0,01^{* \star}$ & $0,62^{\mathrm{a}} \pm 0,00$ & $0,63^{a} \pm 0,01$ & $0,37^{\mathrm{a}} \pm 0,02$ & $0,25^{\mathrm{a}} \pm 0,002$ & 0,9968 \\
\hline & 2 & $0,66^{a} \pm 0,01$ & $0,59^{\mathrm{a}} \pm 0,00$ & $0,59^{a} \pm 0,01$ & $0,41^{a} \pm 0,02$ & $0,22^{a} \pm 0,002$ & 0,9958 \\
\hline & 3 & $0,34^{b} \pm 0,01$ & $0,26^{b} \pm 0,00$ & $0,25^{b} \pm 0,03$ & $0,75^{b} \pm 0,04$ & $0,46^{b} \pm 0,002$ & 0,9991 \\
\hline & 4 & $0,27^{b} \pm 0,01$ & $0,18^{b} \pm 0,00$ & $0,19^{b} \pm 0,04$ & $0,81^{b} \pm 0,05$ & $0,52^{b} \pm 0,002$ & 0,9994 \\
\hline & 5 & $0,23^{b} \pm 0,01$ & $0,17^{b} \pm 0,00$ & $0,20^{b} \pm 0,07$ & $0,80^{b} \pm 0,04$ & $0,55^{b} \pm 0,002$ & 0,9994 \\
\hline & 6 & $0,23^{b} \pm 0,01$ & $0,17^{b} \pm 0,00$ & $0,19^{b} \pm 0,04$ & $0,81^{b} \pm 0,05$ & $0,62^{b} \pm 0,002$ & 0,9995 \\
\hline & 7 & $0,23^{b} \pm 0,01$ & $0,16^{b} \pm 0,00$ & $0,16^{b} \pm 0,02$ & $0,84^{b} \pm 0,05$ & $0,54^{b} \pm 0,002$ & 0,9994 \\
\hline \multirow{7}{*}{ 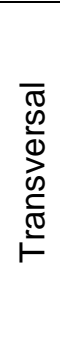 } & 1 & $0,66^{a} \pm 0,07^{* *}$ & $0,58^{a} \pm 0,08$ & $0,59^{a} \pm 0,09$ & $0,41^{a} \pm 0,02$ & $0,24^{a} \pm 0,002$ & 0,9968 \\
\hline & 2 & $0,55^{a b} \pm 0,16$ & $0,47^{a} \pm 0,17$ & $0,51^{\mathrm{ab}} \pm 0,17$ & $0,49^{\mathrm{ab}} \pm 0,03$ & $0,30^{\mathrm{ab}} \pm 0,002$ & 0,9982 \\
\hline & 3 & $0,43^{\mathrm{abc}} \pm 0,05$ & $0,35^{\mathrm{b}} \pm 0,04$ & $0,36^{\mathrm{ab}} \pm 0,14$ & $0,64^{\mathrm{ab}} \pm 0,03$ & $0,39^{\mathrm{ab}} \pm 0,002$ & 0,9989 \\
\hline & 4 & $0,38^{\mathrm{bc}} \pm 0,08$ & $0,24^{b} \pm 0,07$ & $0,25^{b} \pm 0,03$ & $0,75^{\mathrm{b}} \pm 0,03$ & $0,47^{b} \pm 0,002$ & 0,9994 \\
\hline & 5 & $0,41^{b c} \pm 0,08$ & $0,25^{b} \pm 0,07$ & $0,24^{b} \pm 0,01$ & $0,76^{b} \pm 0,05$ & $0,50^{b} \pm 0,002$ & 0,9991 \\
\hline & 6 & $0,27^{\mathrm{bc}} \pm 0,13$ & $0,20^{b} \pm 0,10$ & $0,22^{b} \pm 0,09$ & $0,78^{b} \pm 0,04$ & $0,57^{b} \pm 0,002$ & 0,9995 \\
\hline & 7 & $0,30^{\mathrm{C}} \pm 0,12$ & $0,24^{\mathrm{b}} \pm 0,10$ & $0,25^{\mathrm{b}} \pm 0,04$ & $0,75^{\mathrm{b}} \pm 0,04$ & $0,54^{\mathrm{b}} \pm 0,002$ & 0,9995 \\
\hline
\end{tabular}

Tabla 6: Parámetros del modelo de Peleg y de Bellidio- Hatcher (modelo de Maxwell) a diferentes EM y sentido de carga (SC) para plátano ( ${ }^{* *}$ Letras diferentes indican diferencias significativas en comparación de medias para EM).

\begin{tabular}{|c|c|c|c|c|c|c|c|}
\hline SC & EM & $S^{*}$ & $\mathrm{P}^{*}$ & $\mathrm{Ea}$ & a & b & $\mathrm{R}^{2}$ \\
\hline \multirow{7}{*}{ 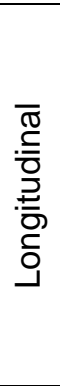 } & 1 & $0,78^{\mathrm{a}} \pm 0,00^{* \star}$ & $0,70^{\mathrm{a}} \pm 0,01$ & $0,71^{a} \pm 0,01$ & $0,29^{\mathrm{a}} \pm 0,01$ & $0,17^{\mathrm{a}} \pm 0,01$ & 0,9949 \\
\hline & 2 & $0,77^{\mathrm{a}} \pm 0,00$ & $0,70^{a} \pm 0,02$ & $0,71^{a} \pm 0,01$ & $0,29^{a} \pm 0,01$ & $0,20^{a} \pm 0,01$ & 0,9955 \\
\hline & 3 & $0,54^{b} \pm 0,01$ & $0,47^{b} \pm 0,02$ & $0,47^{b} \pm 0,02$ & $0,53^{\mathrm{b}} \pm 0,02$ & $0,40^{b} \pm 0,04$ & 0,9989 \\
\hline & 4 & $0,54^{b} \pm 0,01$ & $0,47^{b} \pm 0,02$ & $0,47^{b} \pm 0,01$ & $0,53^{b} \pm 0,01$ & $0,36^{b} \pm 0,02$ & 0,9987 \\
\hline & 5 & $0,53^{\mathrm{b}} \pm 0,00$ & $0,46^{\mathrm{b}} \pm 0,01$ & $0,45^{\mathrm{b}} \pm 0,01$ & $0,55^{\mathrm{b}} \pm 0,01$ & $0,40^{b} \pm 0,05$ & 0,9989 \\
\hline & 6 & $0,51^{b} \pm 0,02$ & $0,44^{b} \pm 0,03$ & $0,44^{b} \pm 0,03$ & $0,56^{b} \pm 0,03$ & $0,44^{b} \pm 0,03$ & 0,9992 \\
\hline & 7 & $0,49^{b} \pm 0,07$ & $0,42^{\mathrm{b}} \pm 0,06$ & $0,42^{b} \pm 0,05$ & $0,58^{b} \pm 0,05$ & $0,38^{b} \pm 0,04$ & 0,9987 \\
\hline \multirow{7}{*}{ 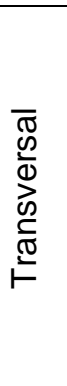 } & 1 & $0,73^{\mathrm{a}} \pm 0,02^{* *}$ & $0,65^{\mathrm{a}} \pm 0,01$ & $0,65^{a} \pm 0,02$ & $0,35^{\mathrm{a}} \pm 0,02$ & $0,23^{\mathrm{a}} \pm 0,03$ & 0,9966 \\
\hline & 2 & $0,75^{a} \pm 0,01$ & $0,67^{a} \pm 0,01$ & $0,68^{a} \pm 0,01$ & $0,32^{a} \pm 0,01$ & $0,20^{a} \pm 0,01$ & 0,9955 \\
\hline & 3 & $0,50^{b} \pm 0,01$ & $0,43^{b} \pm 0,02$ & $0,43^{b} \pm 0,01$ & $0,57^{\mathrm{b}} \pm 0,01$ & $0,41^{b c} \pm 0,02$ & 0,9989 \\
\hline & 4 & $0,49^{b} \pm 0,05$ & $0,40^{\mathrm{b}} \pm 0,06$ & $0,40^{\mathrm{b}} \pm 0,05$ & $0,60^{b} \pm 0,05$ & $0,36^{b} \pm 0,03$ & 0,9985 \\
\hline & 5 & $0,48^{b} \pm 0,01$ & $0,40^{b} \pm 0,02$ & $0,40^{b} \pm 0,02$ & $0,60^{b} \pm 0,02$ & $0,37^{b c} \pm 0,03$ & 0,9987 \\
\hline & 6 & $0,48^{b} \pm 0,02$ & $0,41^{b} \pm 0,02$ & $0,40^{\mathrm{b}} \pm 0,03$ & $0,60^{b} \pm 0,03$ & $0,43^{\mathrm{C}} \pm 0,02$ & 0,9992 \\
\hline & 7 & $0,47^{b} \pm 0,02$ & $0,38^{\mathrm{b}} \pm 0,04$ & $0,38^{b} \pm 0,03$ & $0,62^{\mathrm{b}} \pm 0,03$ & $0,37^{b c} \pm 0,02$ & 0,9988 \\
\hline
\end{tabular}

\section{CONCLUSIONES}

El modelo de Maxwell modificado describe el comportamiento viscoelástico del mango, papaya y plátano más consistentemente que el modelo de Peleg. Para los diferentes estados de madurez, el mango variedad puerco y corazón presenta el mismo comportamiento viscoelástico, mientras que para la papaya variedad Hawaiana y el plátano, el comportamiento elástico disminuye con el estado de madurez. El comportamiento viscoelástico del plátano, determinado con sentido de carga en dirección longitudinal es diferente que en sentido transversal indicando una alta presencia anisotrópica, mientras que la papaya variedad Hawaiana, no presentó diferencias 
significativas, indicando presencia isotrópica. Los parámetros $\mathrm{S}^{*}$ y $\mathrm{P}^{*}$ fueron no sólo mejores discriminadores del comportamiento de relajación que a y b, sino más favorable a la interpretación física.

\section{REFERENCIAS}

Agronet, Análisis - Estadísticas. Ministerio de Agricultura y desarrollo Rural, República de Colombia. http://www.agronet.gov.co. Acceso: 4 de Abril (2011).

Andrés, S.C., N.E. Zaritzky y A.N. Califano, Stress relaxation characteristics of low-fat chicken sausages made in Argentina, Meat Science, 79 (3), 589-594 (2008).

Bellido, G. y D. Hatcher, Asian noodles: Revisiting Peleg analysis for presenting stress relaxation data in soft solid foods, Journal of Food Engineering, 92 (1), 29-36 (2009).

Casierra, F. y O. Aguilar, Calidad en frutos de tomate (Solanum lycopersicum L.) cosechados en diferentes estados de madurez, Agronomía Colombiana, 26 (2), 300-307 (2008).

Ciro, H., F. Monroy y E. Cortés, Estudio preliminar del comportamiento reologico bajo compresión unidireccional de la pulpa de plátano (Musa AAB Simmonds), Revista Dyna, 75 (155), 155-161 (2008).

Ciro, H. y J. Osorio, Avance experimental de la ingeniería de postcosecha de frutas colombianas: resistencia mecánica para frutos de uchuva (Physalis peruviana I), Revista Dyna, 75 (154), 39-46 (2008).

Ciro, H., D. Vahos y C. Márquez C, Estudio experimental de la fuerza de Fractura en frutas tropicales: el tomate de Árbol (Cyphomandra betacea Sendt), Revista Dyna, 72 (146), 55-64 (2005).

Dadzie, B. y J. Orchard, Evaluación rutinaria postcosecha de híbridos de bananos y plátanos: criterios y métodos. Guías técnicas INIBAP 2. Instituto Internacional de Recursos Fitogenéticos 2: 76 (1997).

FAO. Situación actual y perspectivas a plazo medio para las frutas tropicales. Organización de las naciones unidas para la agricultura y la alimentación.

http://www.fao.org/es/esc/common/ecg/218/es/Sit_web_s.pdf. Acceso: 3 de Abril (2012).

FAO. Pérdidas en la manipulación después de la cosecha, grupo intergubernamental sobre el banano y las frutas tropicales (Cuarta reunión). FAO, Guayaquil, p 2-3 (2005).

Hatcher, D., G. Bellido, J. Dexter, M. Anderson y B. Fu, Investigation of uniaxial stress relaxation parameters to characterize the texture of yellow alkaline noodles made from durum and common wheats, Journal of Texture Studies, 39 (6), 695-708 (2008).

Hatcher, D., J. Dexter y B. Fu, Refrigerated storage of yellow alkaline durum noodles: impact on color and texture, Cereal Chemistry, 86 (1), 106-112 (2009).

Linares, A.R., M.L. Vergara y S.L. Hase, Efecto de la cocción sobre los parámetros texturales de dos variedades de mandioca, Información Tecnológica, 16 (5), 3-9 (2005).

Melgosa, M., M. Pérez, A. Yebra, R. Huertas y E. Hita, Algunas reflexiones y recientes recomendaciones internacionales sobre evaluación de diferencias de color, Óptica pura y aplicada, 34, 4-10 (2001).

Moretti, C. y S. Sargent, Alteração de sabor e aroma em tomates causado por impacto, Scientia Agricola, 57 (3), 385-388 (2000). 
OriginLab Corporation. OriginPro 8 SR0 versión 8.072., http://www.OriginLab.com. Acceso: 4 de Abril (2011).

Ospina, D., H. Ciro y I. Aristizábal, Determinación de la fuerza de la fractura superficial y fuerza de firmeza en frutas de lulo (Solanum quitoense x Solanum hirtum), Revista Facultad Nacional de Agronomía, 60 (2), 4163-4178 (2007).

Peleg, M y M. Normand, Comparison of two methods for stress relaxation data presentation of solid foods, Rheologica Acta, 22 (1), 108-113 (1983).

Peña, M., Tratamiento cuarentenario contra la mosca mediterráneo (Ceratitis capitata) en mangos variedad Haden (Manguífera índica) con irradiación gamma (Co-60). Magíster en Ciencia de los Alimentos, Universidad Nacional Mayor de San Marcos, Lima (2008).

Rao, M., S. Syed, S. Rizvi, y A. Datta, Engineering Properties of Foods, 41-113, Taylor \& Francis Group, New York, Estados Unidos (2005).

Santamaría, F., E. Sauri, F. Espadas, R. Díaz, A. Larqué y J. Santamaría, Postharvest ripening and maturity indices for maradol papaya, Interciencia, 34 (8), 583- 588 (2009).

Singh, H., A. Rockall, C.R. Martin, O.K. Chung y G.L. Lookhart, The analysis of stress relaxation data of some viscoelastic foods using a texture analyzer, Journal of Texture Studies, 37 (4), 383392 (2006).

StatSoft, Inc. STATISTICA, versión 7. http://www.statsoft.com. Acceso: 4 de Abril (2011). 